\title{
Inactivation of auxin in tobacco transformed with the indoleacetic acid-lysine synthetase gene of Pseudomonas savastanoi
}

\author{
Charles P. Romano, Mich B. Hein, ${ }^{1}$ and Harry J. Klee ${ }^{2}$ \\ Monsanto Company, St. Louis, Missouri 63198 USA; ${ }^{1}$ Research Institute of Scripps Clinic, La Jolla, California 92037 USA
}

The iaal gene of Pseudomonas syringae, subspecies savastanoi, encodes an indoleacetic acid (IAA)lysine synthetase. To determine the effects of converting IAA to IAA-lysine in whole plants, the iaaL gene was fused to a constitutive plant promoter and introduced into tobacco plants. Biochemical analyses show that endogenous IAA is reduced by up to 19-fold in iaaL plants. Tobacco plants expressing the iaaL gene display reduced apical dominance, reduced rooting, and inhibition of vascular differentiation. The phenotypic effects of iaaL gene expression are reverted by crossing iaaL plants with plants that overproduce IAA. These data indicate that iaaL can act as an anti-auxin gene in vivo and confirm the role of auxin in the control of apical dominance, root growth, and vascular differentiation in whole plants.

[Key Words: Auxin; IAA conjugates; plant development]

Received September 11, 1990; revised version accepted December 27, 1990.

Indoleacetic acid (IAA) is one of several phytohormones that collectively coordinate the growth and differentiation of plant cells. Diverse processes such as shoot gravitropism, maintenance of apical dominance, and differentiation of vascular tissue all appear to be dependent on IAA (i.e., auxin) (Kaufman and Song 1987; Tamas 1987; Aloni 1988). Control of plant growth and development is presumably mediated by the demonstrated effects of IAA on cellular processes such as cell wall extensibility, cell division, and gene expression (Cleland 1987; Hagen 1987). While IAA has clearly been implicated in the regulation of these and other aspects of plant development, many critical questions remain. For example, in many instances the relative importance of absolute IAA levels versus the sensitivity of the responding target tissue are not clear (Trewavas 1982). The biochemical mechanisms by which a plant maintains optimal IAA levels are also not fully understood (Bandurski 1984). Furthermore, the counteracting influence of cytokinins on auxin activity is well documented but not mechanistically understood (Skoog and Miller 1957).

To study phytohormone action, plants have been transformed with genes that modulate phytohormone levels or responses (Klee et al. 1987; Schmülling et al. 1988; Medford et al. 1989). In contrast to the classical technique of phytohormone application, endogenous production in transgenic plants is not complicated by uptake, transport, wounding, or tissue culture phenom-

${ }^{2}$ Corresponding author. ena. Deliberate manipulation of phytohormone levels in transgenic plants thus permits direct analysis of the role of phytohormone levels in whole plants. Furthermore, the role of phytohormones in controlling developmental processes occurring under certain temporal, spatial, or environmental conditions can also be tested by placing phytohormone-modulating genes under the control of appropriate transcriptional promoters.

To understand the roles and interactions of phytohormones in plant growth and development, the effects of both increased and decreased hormone levels must be examined. While the effects of increased IAA and cytokinin levels have been studied in whole transgenic plants (Klee et al. 1987; Medford et al. 1989), complementary studies of plants with phytohormone deficiencies have not been reported. To decrease hormone levels in transgenic plants, genes that irreversibly inactivate hormones must first be identified. In this study, the iaaL gene of Pseudomonas syringae subspecies savastanoi $\{P$. savastanoi) was tested for anti-auxin activity in transgenic tobacco. The iaaL locus encodes an IAA-lysine synthetase activity that converts IAA to $\mathrm{N}^{\epsilon}$-indole3-acetyl-L-lysine or IAA-lysine (Glass and Kosuge 1986; Hutzinger and Kosuge 1968b; Roberto et al. 1990). Conjugation of IAA with aspartate or glutamate appears to play a crucial role in the inactivation and storage of endogenous IAA pools in plants (Cohen and Bialek 1984). Thus, expression of the iaaL gene in plants might reduce the levels of endogenous IAA. Moreover, because IAAlysine is not ordinarily found in plants, it may not be 
efficiently converted to IAA by endogenous plant enzymes (Cohen and Bandurski 1982). Here we demonstrate that iaaL functions as an anti-auxin gene when expressed in tobacco. Finally, the previously undescribed effects of IAA depletion on the growth and development of whole plants are examined.

\section{Results}

\section{Expression of iaaL in plants}

As the iaaL locus of $P$. savastanoi is ordinarily part of a polycistronic, bacterial mRNA (Roberto et al. 1990), it was first necessary to place the gene in a plant expression vector. The iaaL structural gene and a nopaline synthetase (NOS) polyadenylation signal were transcriptionally fused to the cauliflower mosaic virus (CaMV) 35S promoter. While the CaMV $35 \mathrm{~S}$ promoter drives high levels of reporter gene expression in leaves, stems, and roots throughout plant development, its activity varies in different cell types within those organs (Jefferson et al. 1987; Benfey and Chua 1989). The chimeric gene was placed in a disarmed T-DNA vector with a neomycin phosphotransferase (nptII) selection marker and introduced into Nicotiana tabacum subspecies samsun by Agrobacterium tumefaciens-mediated transformation (Horsch et al. 1985). Transformed $\mathrm{R}_{0}$ plants were regenerated on media with standard levels of phytohormones from kanamycin-resistant callus tissue (Horsch et al. 1985). Expression of the 35S-iaaL gene at the levels observed in the transgenic lines did not appear to interfere with efficient plant regeneration. Nonetheless, it is possible that T-DNA insertion events resulting in higher levels of iaaL expression may inhibit plant regeneration, thus preventing recovery and analysis. All analyses were performed on $R_{1}$ or $R_{2}$ progeny of the $R_{0}$ plants containing the T-DNA insert|s|.

To determine whether enzymatically active iaaL protein is produced in transformed plants, extracts from transformed and untransformed leaves were assayed for IAA-lysine synthetase activity (Fig. 1). Thin-layer chromatographs show that extracts from 35S-iaaL plants yield an enzymatic reaction product indistinguishable from the authentic IAA-lysine produced by bacterially derived iaaL protein (Roberto et al. 1990). Wild-type plant extracts have no detectable iaaL activity. Plants containing the $35 \mathrm{~S}-$ iaaL gene thus appear to produce enzymatically active $i a a L$ protein.

Quantitation of IAA content in $35 \mathrm{~S}-\mathrm{iaaL}$ and 19S-iaaM plants

Levels of free (unconjugated) IAA in several independent 35S-iaaL lines, wild-type, and one CaMV 19S-iaa tryptophan monooxygenase (19S-iaaM) line were determined as described (Table 1; Materials and methods). The 19S-iaaM plant contains a previously described fusion of the CaMV 19S promoter to the tryptophan monooxygenase structural gene (iaaM) that increases IAA levels (Klee et al. 1987). Free IAA levels are reduced by an

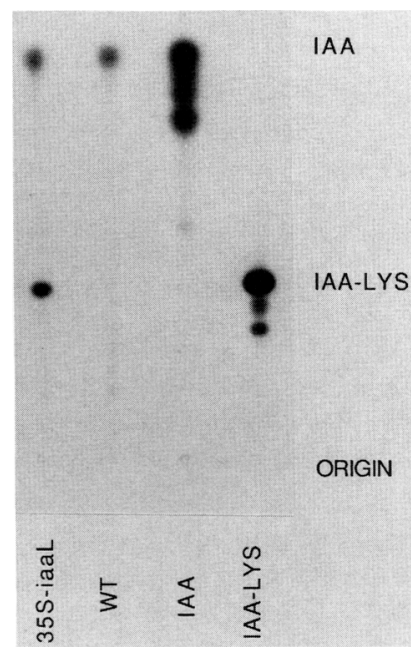

Figure 1. Presence of iaaL activity in 35S-iaaL plant extracts. Extracts from 35S-iaaL leaves, wild-type (WT) leaves, and bacteria-expressing iaaL (IAA-LYS) were assayed for conversion of the ${ }^{3} \mathrm{H}$-labeled IAA substrate to IAA-lysine. Reaction products were purified, subjected to thin-layer chromatography, and fluorographed as described (Materials and methods). ${ }^{3} \mathrm{H}$-labeled IAA substrate (IAA) was also included as a standard.

average of fivefold in the $35 \mathrm{~S}-$ iaa $L$ plants examined relative to the wild-type control. In 35S-iaaL extracts, a large UV-absorbing peak co-elutes with an authentic standard of bacterially produced IAA-lysine on high-performance liquid chromatography (HPLC). The amount of IAA represented by this fraction greatly exceeds the ob-

Table 1. Phenotype, iaal-specific activities, and free IAA levels in wild-type, 19S-iaaM, and 35S-iaaL plants

\begin{tabular}{|c|c|c|c|c|}
\hline \multirow[b]{2}{*}{ Line $^{a}$} & \multirow[b]{2}{*}{ Phenotype $^{\mathrm{b}}$} & \multirow[b]{2}{*}{$\begin{array}{l}\text { Sp. } \\
\text { act. }^{c}\end{array}$} & \multicolumn{2}{|c|}{ IAA levels ${ }^{d}$} \\
\hline & & & levels & $\begin{array}{l}\text { fold } \\
\text { difference }\end{array}$ \\
\hline Wild type & - & 0 & $13.2 \pm 3.1$ & - \\
\hline 19S-iaaM 1 & - & - & $95.1 \pm 2.8$ & $(7.3)$ \\
\hline $35 \mathrm{~S}-$ iaaL 1 & weak & 5 & $5.4 \pm 2.0$ & 2.5 \\
\hline $35 S-i a a L 2$ & moderate & 18 & $3.0 \pm 2.4$ & 4.4 \\
\hline $35 S-i a a L 3$ & severe & 39 & $1.7 \pm 1.1$ & 7.8 \\
\hline $35 S-i a a L 4$ & severe & 52 & $2.8 \pm 0.8$ & 4.7 \\
\hline 35S-iaaL 5 & severe & 110 & $1.3 \pm 1.1$ & 10 \\
\hline $35 S-i a a L 6$ & severe & 120 & $0.7 \pm 0.6$ & 19 \\
\hline $35 \mathrm{~S}-\mathrm{iaaL} 7$ & severe & 24 & $3.1 \pm 1.2$ & 4.3 \\
\hline
\end{tabular}

${ }^{a}$ Line numbers 35 -iaal 1-7 correspond to plants and leaves shown in all figures and throughout text.

bLeaf-wrinkling phenotype only (for corresponding specimens, see Fig. 2b).

(U/min per mg of protein); units are pmoles of IAA converted to IAA-lysine.

dIAA levels are in $\mathrm{ng} / \mathrm{g}$ tissue fresh weight; means of two independent determinations of each extract \pm the S.E. measurement are shown. The parenthesized fold difference for 19S-iaaM represents the fold increase relative to wild type; all other values represent the fold decrease relative to wild type. 
served difference in free IAA concentrations between wild-type and 35S-iaaL plants. In the 19S-iaaM plant, free IAA levels are about sevenfold higher than in the wild-type control. These data thus show that both the iaaL and iaaM gene products, respectively, reduce and increase IAA levels in vivo.

\section{Phenotypic consequences of 35S-iaaL expression}

Effects of iaaL expression were examined in $R_{1}$ progeny of 18 independent 35S-iaaL lines. Seedlings are essentially wild type in appearance. However, the $35 \mathrm{~S}-$ iaaL plants display characteristic growth defects as they mature. First, the true leaves emerge and become wrinkled as they expand. In juvenile plants, the leaves become increasingly wrinkled with distance from the apex (Fig. 6, below; see 35S-iaaL). At maturity, severe leaf wrinkling and loss of apical dominance are clearly evident in $35 \mathrm{~S}$-iaaL plants (Fig. 2a). Nonetheless, flowering is just slightly delayed in the fully fertile $35 \mathrm{~S}$-iaaL plants relative to wild-type plants (Table 2). Finally, the height and internode length of $35 \mathrm{~S}-$ iaaL plants are essentially the same as for wild-type plants (Table 2).

Apical dominance is also significantly reduced in flowering 35S-iaaL plants relative to wild-type control plants at the same stage. The primary difference is that axillary bud growth is far more extensive in the 35S-iaaL plants (Fig. 3). However, growth of both wild-type and 35S-iaaL axillary buds is inhibited until the vegetative to floral transition occurs. Because wild-type and 35S-iaaL axillary buds start growing at the same time, buds of $35 \mathrm{~S}-$ iaaL plants must become larger by growing at a greater rate. This phenotype is very similar to that observed in
Table 2. Comparisons of wild-type and $35 S$-iaaL plants

\begin{tabular}{|c|c|c|c|c|}
\hline & Wild type & $35 \mathrm{~S}-\mathrm{iaaL} 4$ & $35 \mathrm{~S}-i a a L 6$ & $35 S-i a a L 7$ \\
\hline $\begin{array}{l}\text { Flowering } \\
\text { time (days) }\end{array}$ & $58 \pm 0$ & $63.0 \pm 2.4$ & $63.4 \pm 2.6$ & $61.1 \pm 1.9$ \\
\hline Height $(\mathrm{cm})$ & $69 \pm 2.7$ & $66 \pm 2.8$ & $70 \pm 3.4$ & NT \\
\hline Internode & & & & \\
\hline length $(\mathrm{cm})$ & $2.5 \pm 0.1$ & $2.2 \pm 0.2$ & $2.3 \pm 0.3$ & NT \\
\hline length $(\mathrm{cm})$ & $23.1 \pm 1.1$ & $12.4 \pm 0.8$ & $15.1 \pm 1.8$ & $17.2 \pm 1.7$ \\
\hline
\end{tabular}

Mean values and standard deviations for the indicated plant growth parameters are shown. Data for each genotype was derived from a minimum of six plants. (NT) Not tested. The $\mathrm{R}_{2}$ transgenic plants were homozygous for the 35S-iaaL T-DNA insert(s). Experimental details are described in Materials and methods.

transgenic tobacco with increased cytokinin levels (Medford et al. 1989).

Rooting inhibition is also observed in 35S-iaaL plants (Fig. 4). Individual roots of the 35S-iaaL plants are as long as those of wild-type plants but are much thinner. Inhibition of rooting in the $35 \mathrm{~S}-$ iaaL plants is only evident at later stages of development, as no significant differences in the extent of growth or the number of lateral roots is observed in 18-day-old seedlings /data not shown). Rooting inhibition also occurs in transgenic tobacco with elevated cytokinin levels (Medford et al. 1989). However, rooting of tobacco with excess cytokinin is inhibited at the seedling stage.

The most striking phenotype of the 35S-iaaL tobacco plants is the extremely wrinkled growth habit of the leaves (Fig. 2b). Wrinkling becomes more severe as the leaves age and become more distant from the apex, re-

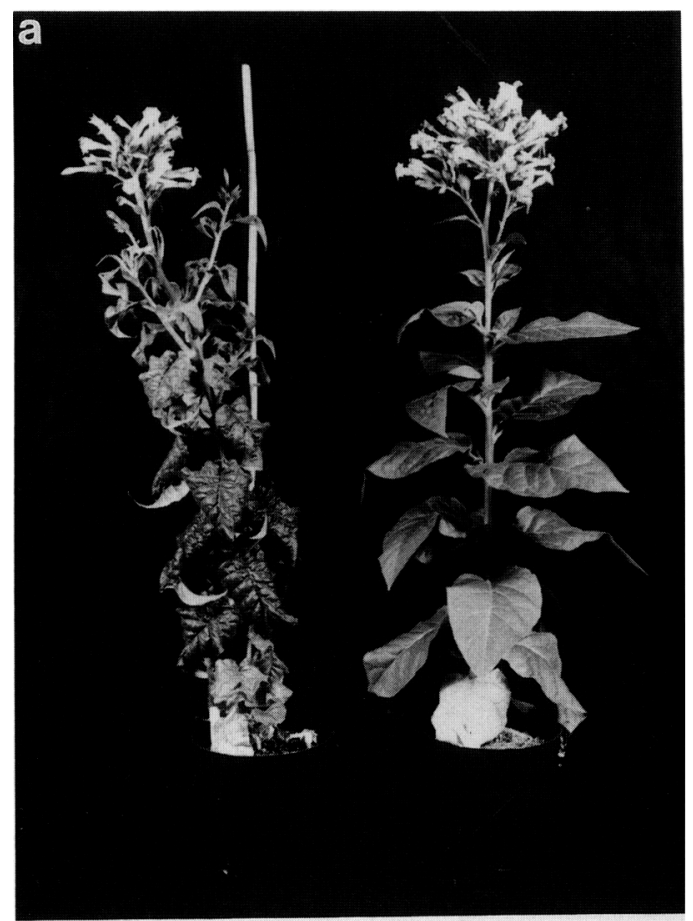

35S-iaaL

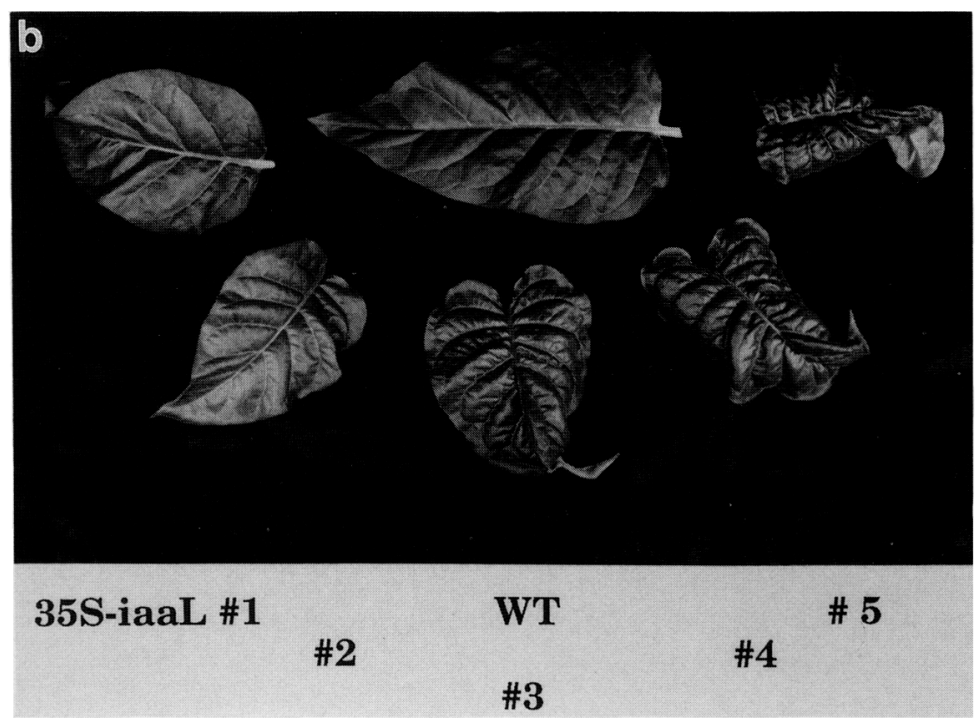

Figure 2. (a) Comparison of a mature 35S-iaaL with a wild-type (WT) plant. The $35 \mathrm{~S}-$ iaaL plant shown is line 4 in Table 1 and is representative of the 18 independent 35S-iaaL lines examined. $(b)$ Comparison of leaves from wildtype and five independent $35 \mathrm{~S}-$ iaa $L$ lines. Leaves are from the 12 th node from the base of a mature plant. The wild-type leaf is at center and the 35S-iaaL leaves are in a semicircle with line 1 at the left and line 5 at right. Line numbers correspond to those in Tables 1-3. 

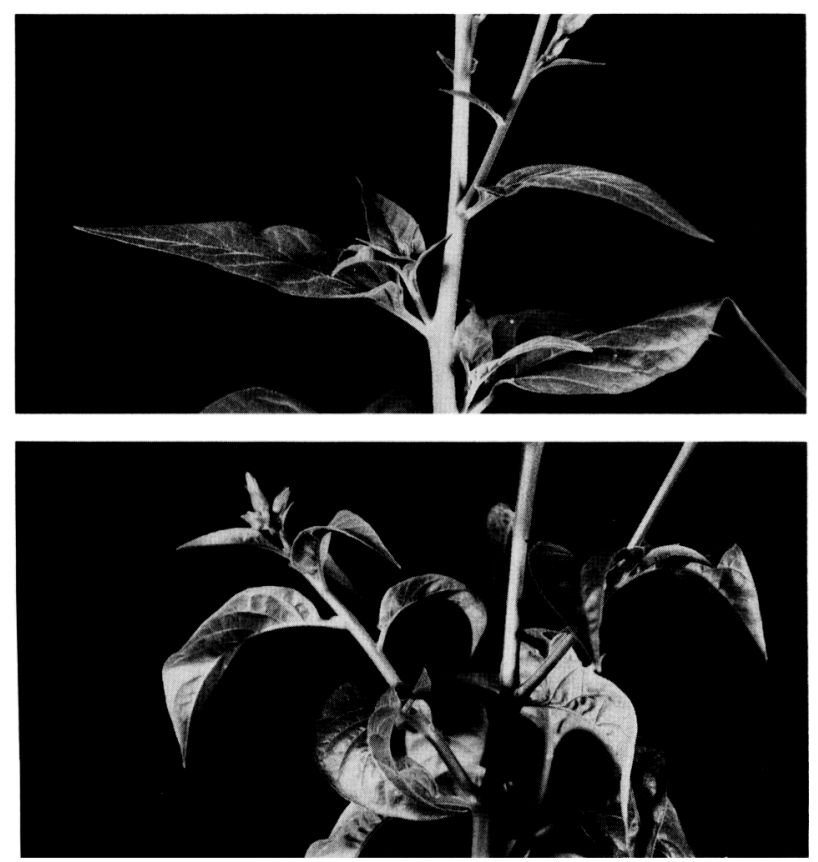

Figure 3. Comparison of axillary buds of mature 35S-iaa $L$ and wild-type plants. Axillary buds at the 21 st node from the base of a wild-type (top) or 35S-iaaL line 7 (bottom) plant are shown.

flecting the fact that endogenous auxin levels are highest in the new leaves and decline as the leaf ages /Schneider and Wightman 1978). No obvious changes in the sizes, types, or organization of cell layers within $35 \mathrm{~S}-i a a L$ leaves were apparent in observations of thin sections. Development of the vasculature may lag behind the division and expansion of the remaining cells of the 35SiaaL leaves, resulting in the wrinkled phenotype. Midrib length in fully expanded 35S-iaaL leaves is reduced by up to $50 \%$ relative to wild-type controls (Table 2), supporting the notion that wrinkling is caused by inhibition of vascular development. A similar leaf-wrinkling phenotype also results from expression of the Agrobacterium rhizogenes rolA gene in transgenic tobacco (Schmülling et al. 1988; Sinkar et al. 1988).

Vascular development is inhibited in stems of the 35S-iaaL tobacco (Fig. 5). Transformed plants have fewer and larger xylem elements than the wild-type control plants. Lignin deposition, visualized by phloroglucinol staining, is considerably reduced in the xylem of $35 \mathrm{~S}-$ iaaL plants, accounting for the observed thinning of the xylem cell walls (Fig. 5). These effects are consistent with the postulated role of auxin in xylogenesis (Aloni 1988) and once again similar to what occurs in transgenic plants with increased cytokinin levels (Medford et al. 1989).

Specific iaal activity levels, phenotypic effects, and IAA levels in independent $35 \mathrm{~S}-\mathrm{iaaL}$ lines

To determine whether there is a correlation between $35 \mathrm{~S}$-iaaL expression levels, severity of phenotypic ef- fects, and IAA content, a linear assay for iaaL activity was devised and used to compare the specific activity levels in leaves of several independent transgenic lines (see Materials and methods; Table 1). Specific activity levels differ by as much as 25 -fold in the lines examined. These differences are presumably due to the chromosomal position effects commonly observed in gene transfer experiments (Fraley et al. 1986). There is a reasonably close correlation between iaaL specific activity levels and the severity of the leaf-wrinkling phenotype (Fig. 2b; Table 1, lines 1-5). Finally, the iaaL specific activity levels in leaves also show some correlation with the IAA levels measured in whole juvenile plants. Lines that display the highest levels of $i a a L$ gene expression have the greatest reductions in IAA levels while the line with the lowest level of iaaL gene expression also has the lowest reduction in IAA levels (Table 1 , lines 5, 6, 1, respectively). However, there is not an exact correlation between specific activity levels and IAA content in lines with intermediate iaaL gene expression levels (Table 1, lines $2,3,4,7)$. Lack of an absolute correlation between

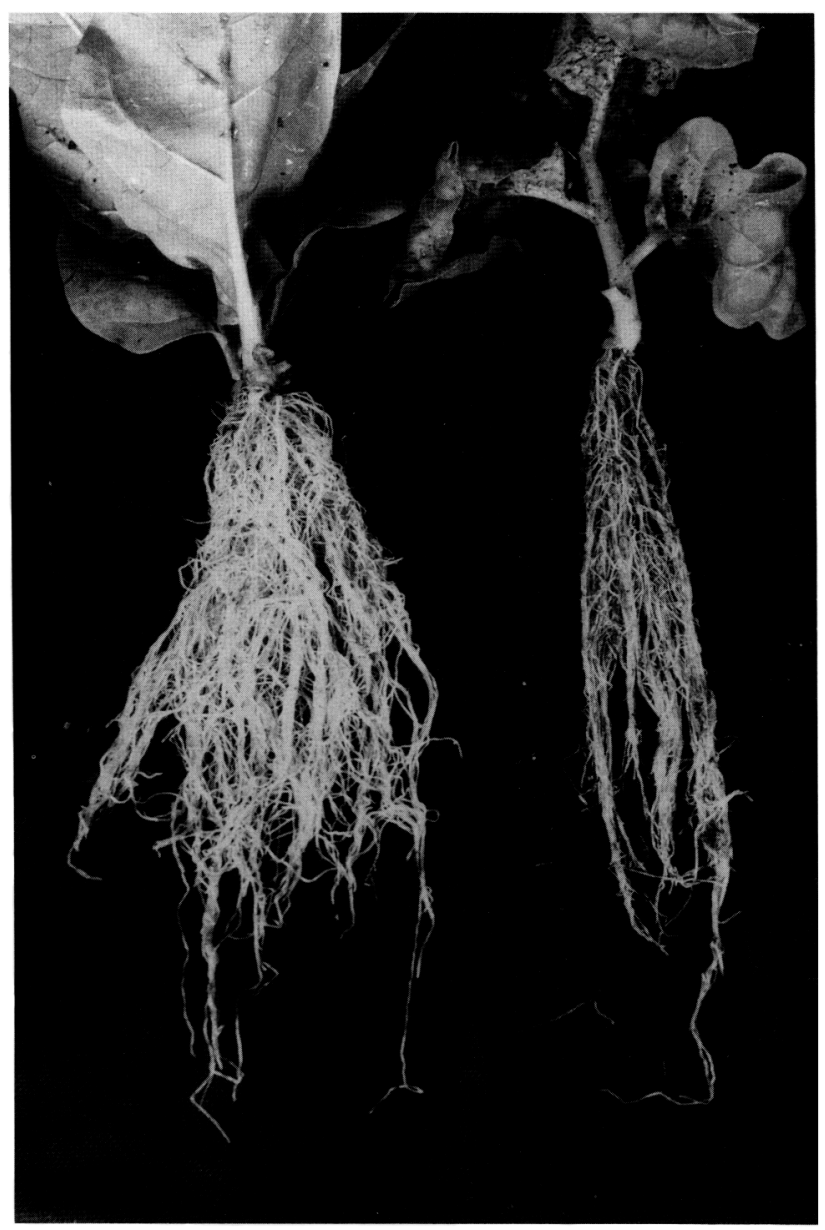

Figure 4. Comparison of root systems of 35S-iaaL and wildtype plants. Root systems are of vegetative stage wild-type (left) and $35 \mathrm{~S}$-iaaL line 4 (right) plants grown in 4 -inch pots. Soil was washed from the roots with water. 


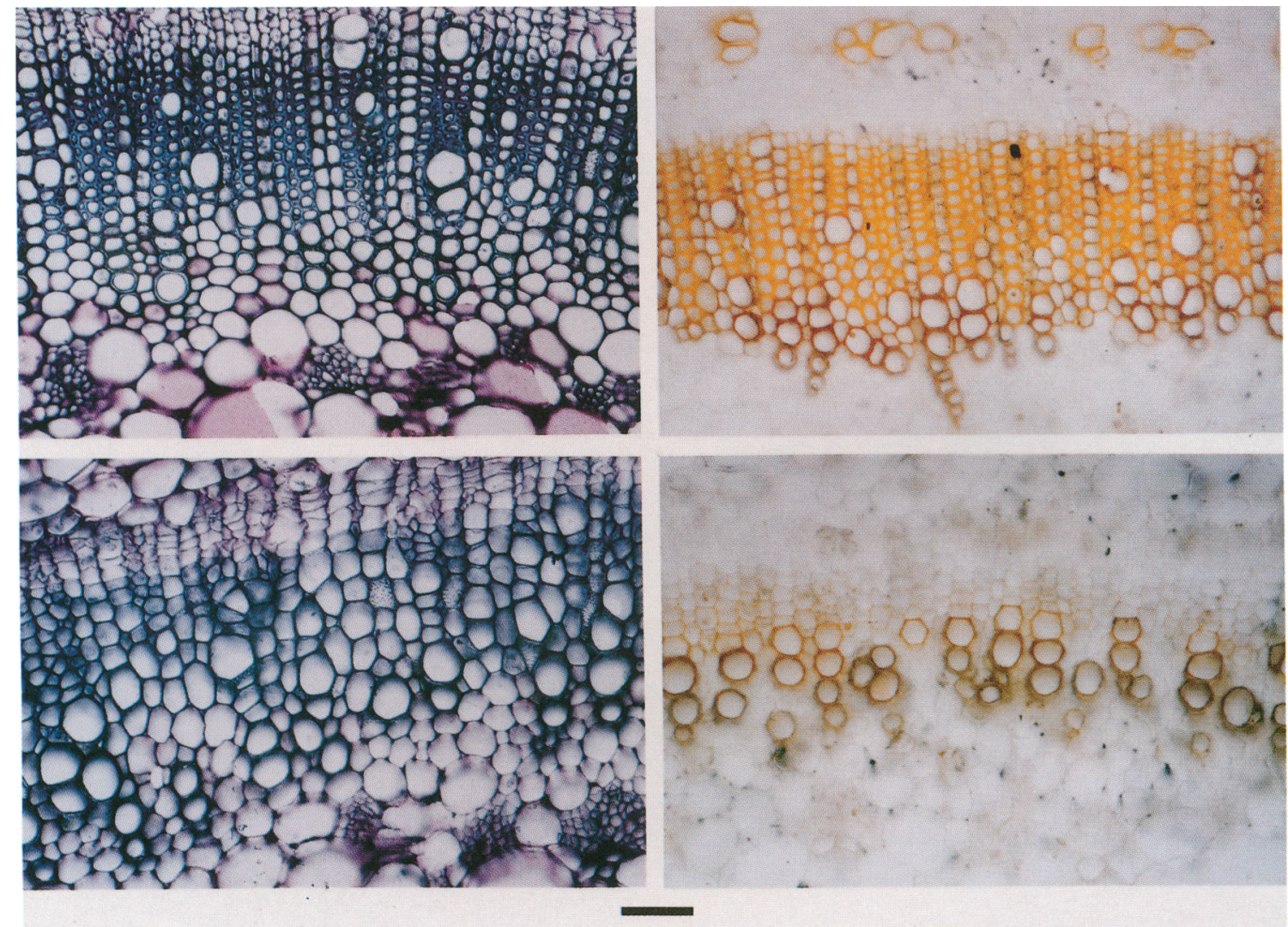

Figure 5. Cross sections (100-mm thick) of stems of wild-type and 35S-iaaL plants. Wild-type stem sections are at top and 35S-iaaL line 2 sections are at bottom. Sections from the 4th node from the base of a mature plant were prepared as described in Materials and methods and stained with either toluidine blue (left) or phloroglucinol (right). Bar, $100 \mathrm{~mm}$.

the leaf iaaL expression levels and whole plant IAA levels is not surprising as the tissue specificity of CaMV $35 \mathrm{~S}$ expression often varies in independent transgenic lines (Benfey and Chua 1989).

Reversion of the iaaL phenotypes by iaaM-mediated auxin overproduction

At least two models can be advanced to account for the phenotypic effects of iaaL gene expression. The simplest explanation is that the effects are due to reductions in endogenous IAA levels. Alternatively, the effects might be due to an activity of the novel IAA-lysine conjugate produced by iaaL. If the effects are due to a decrease in IAA levels, they should be reversed by increasing IAA levels in 35S-iaaL plants. However, if the effects are due to an activity of the IAA-lysine conjugate, they would be unaffected or perhaps even exacerbated by increasing IAA levels in 35S-iaaL plants.

To distinguish between these two possibilities, four independent 35S-iaaL lines homozygous for the T-DNA insert(s) were crossed to a 19S-iaaM line homozygous for its insert(s). Like petunia, tobacco transformed with the 19S-iaaM gene display classic symptoms of auxin overproduction such as increased apical dominance and adventitious rooting (Klee et al. 1987; data not shown). The 35 -iaaL lines 3, 4, 6, and 7 with 4-, 5-, 19-, and 4-fold reduced IAA levels, respectively, were crossed to a 19SiaaM line with 7-fold increased IAA levels (Table 1). Progeny of the cross have nearly normal leaves and apical dominance, demonstrating that decreases in IAA levels are primarily responsible for the $35 \mathrm{~S}-$ iaa $L$ phenotype (Fig. 6). Southern and Northern blot analyses of the $F_{1}$ plants also indicate that both genes are present and expressed, eliminating the possibility that other mechanisms such as gene suppression are responsible for reversion of the 35S-iaaL phenotype (data not shown; Napoli et al. 1990; van der Krol et al. 1990|. Finally, IAA levels in leaves of $35 \mathrm{~S}-$ iaaL $\times 19 \mathrm{~S}-$ iaaM plants are slightly elevated relative to wild-type plants, reduced relative to the 19S-iaaM parent, and increased relative to the $35 \mathrm{~S}$ iaal parents (Table 3). A chromatographic peak corresponding to the presumed IAA-lysine conjugate is also present in the 35S-iaaL $\times 19 \mathrm{~S}-$ iaaM extracts at levels comparable to that of the $35 \mathrm{~S}-$ iaaL parental extracts (data not shown). Thus, these data biochemically confirm that IAA deficits rather than the presence of IAAlysine are responsible for the $35 \mathrm{~S}-$ iaaL phenotype.

\section{Discussion}

In this study, three observations indicate that the iaaL gene product acts as an anti-auxin by lowering endoge- 

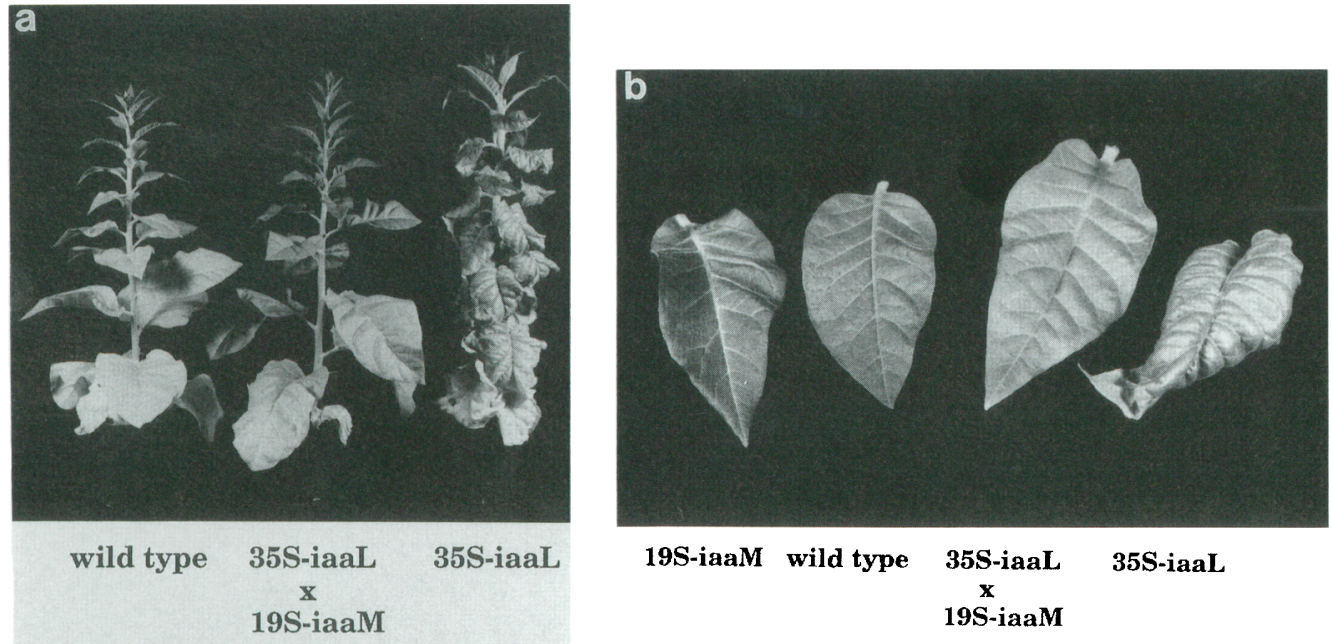

Figure 6. (a) Comparison of wild-type, 35S-iaaL 19S-iaaM, and 35S-iaaL $\times 19 \mathrm{~S}-i a a M$ plants. Progeny from the cross of $35 \mathrm{~S}-i a a L$ line 6 to 19S-iaaM line 1 and the parental 35S-iaaL line 6 are shown. (b) Comparison of wild-type, 35S-iaaL, 19S-iaaM, and $35 \mathrm{~S}-$ iaaL $\times 19 \mathrm{~S}-$ iaaM leaves. Shown are 11 th node leaves from a cross of 35S-iaaL line 4 to $19 \mathrm{~S}-$ iaaM line 1 (35S-iaaL $\times 19 \mathrm{~S}-$ iaaM) and to wild type $(35 S-i a a L)$.

nous IAA levels in transformed tobacco plants. First, direct biochemical analysis shows that the endogenous IAA levels are reduced by up to 19 -fold in $35 \mathrm{~S}$-iaaL plants. Second, 35S-iaaL plants display phenotypic traits (i.e, loss of apical dominance, rooting inhibition, and retardation of vascular development) that are classically observed when plants are deficient or insensitive to auxin (Kelly and Bradford 1986; Estelle and Somerville 1987; Aloni 1988; Lincoln et al. 1990). Third, the effects of $i a a L$ are negated by provision of additional endogenous IAA.

The apparent inactivity of IAA-lysine is consistent with a number of observations. First, aspartate and glutamate conjugates of IAA appear to be inactive storage forms of IAA in legume seeds (Cohen and Bialek 1984; Bialek and Cohen 1989). In fact, an IAA-aspartate conjugation activity appears to be induced by excess auxin in pea (Venis 1972). Second, the auxin activity of IAAamino acid conjugates in bioassays actually results from their hydrolysis to IAA (Hangartner and Good 1981; Bi-

Table 3. IAA levels in $35 S$-iaaL $\times 19 S$-iaaM hybrids

\begin{tabular}{lc}
\hline Plant genotype & IAA levels \\
\hline Wild type & 11 \\
19S-iaaM $1 \times$ wild type & 54 \\
35S-iaaL $4 \times$ wild type & 5 \\
35S-iaaL $4 \times 19$ S-iaaM & 17 \\
35S-iaaL 6 & 1 \\
35S-iaaL $6 \times 19$ S-iaaM & 15
\end{tabular}

IAA levels in nanograms of IAA per gram of fresh weight of tissue are shown. The coefficient of variability of measurement for these analyses was $12 \%$. The $35 \mathrm{~S}-$ iaaL 6 sample was from a plant homozygous for the T-DNA insert(s). alek et al. 1983). Thus, the finding that IAA-lysine has $20-40 \%$ of the activity of IAA in Avena coleoptile bioassays is presumably due to hydrolysis of the conjugate to IAA (Hutzinger and Kosuge 1968a). Finally, structure/ function studies of various auxins also indicate that IAA-lysine should have little auxin activity (Thimann 1963).

The similar rooting inhibition and loss of apical dominance phenotypes observed in transgenic plants with either auxin deficits (this study) or excess cytokinin (Medford et al. 1989) suggests that these processes are controlled by the auxin/cytokinin ratio rather than by the absolute level of either phytohormone. Auxin/cytokinin ratios were first implicated in maintenance of apical dominance and root initiation via classic phytohormone application and tissue culture experiments (Thimann and Skoog 1934; Skoog and Miller 1957). As apical dominance and rooting are also inhibited in 35S-iaaL Arabidopsis plants (C. Romano and H. Klee, unpubl.), this principle probably holds for a variety of plant species. However, measurements of cytokinin levels in auxin-deficient plants and auxin levels in cytokinin-rich plants clearly must be made to definitively prove that these whole plant processes are controlled by the auxin/ cytokinin ratio.

Quantitative differences in cell differentiation are clearly observed in comparisons of the vascular tissue of wild-type and 35S-iaaL plants. The number of differentiated xylem cells and xylem cell wall thickness are reduced in 35S-iaaL plants. These differences between the $35 \mathrm{~S}-$ ia $a$ L and wild-type plants are comparable to differences in vascular tissue derived from auxin-rich apical regions and auxin-poor basal regions of an individual plant (Aloni and Zimmermann 1983). Moreover, overproduction of IAA in transgenic petunia results in xylem 
proliferation while overproduction of cytokinins in transgenic tobacco and Arabidopsis results in the inhibition of xylem formation (Klee et al. 1987; Medford et al. 1989). Taken together, these results support the hypothesis that auxin increases the rate at which the undifferentiated cambial cells divide and enter the xylem differentiation pathway (Aloni 1988). Aloni's hypothesis that auxin controls xylem cell diameter by increasing the degree and rate of cell wall deposition is also supported by these observations. Consequently, current models of the role of auxin in whole plant vascular differentiation based on hormone application experiments are consistent with studies of transgenic plants with genetically engineered hormone imbalances.

It is most interesting that the $i a a L$-mediated decrease in endogenous free IAA circumvents whatever mechanisms ordinarily maintain the IAA pool at an appropriate level. The plant enzymes that synthesize, degrade, and interconvert IAA and its conjugates clearly must be regulated at some level (Bandurski 1984). In contrast with the introduced iaaL activity, endogenous IAA-conjugating enzymes may be induced by conditions of IAA excess or inhibited by excess cytokinin (Venis 1972; Montague et al. 1981). By introducing iaaL into a genetically tractable plant such as Arabidopsis and screening for secondsite mutations that counteract its effects, it may be possible to identify endogenous genes that regulate auxin levels. Further understanding of auxin metabolism might also be gained by targeting iaaL protein to certain tissues or subcellular compartments.

In summary, we demonstrate here that the iaaL locus of $P$. savastanoi functions as an anti-auxin gene when introduced into tobacco under the control of a constitutive plant promoter. Observations of the auxin-deficient plants coupled with previous observations of cytokininoverproducing plants indicate that auxin/cytokinin ratios rather than the absolute level of either phytohormone control apical dominance and root growth. Vascular differentiation is also inhibited in the auxin-deficient plants, supporting previous models of auxin action based on application experiments and correlative studies. Finally, we note that the iaaL gene will be most useful in determining the role of auxin in tissue-specific processes when fused to regulated promoters.

\section{Materials and methods}

Construction of 35S-iaaL (pMON690) and 19S-iaaM (pMON518)

The 35S-iaaL or pMON690 plasmid was constructed by first introducing a BglII site upstream of the NdeI site of pMON686 (Kunkel 1985; Roberto et al. 1990) and then ligating the resultant $1.5-\mathrm{kb}$ BglII-ClaI iaaL fragment into the BglII-ClaI-cut pMON530 CaMV 35S plant expression vector (Rogers et al. 1987). Construction of the 19S-iaaM vector (pMON518) was described previously (Klee et al. 1987). Both pMON690 and pMON518 were mobilized into Agrobacterium that contained pTiT37-SE, a disarmed nopaline-type Ti plasmid, with the pRK2013 helper plasmid (Rogers et al. 1987; Ditta et al. 1980).

\section{Introduction of genes into plants}

Agrobacterium strains containing the indicated vectors were used to transform leaf discs of Nicotiana tabacum cultivar samsun (Horsch et al. 1985). Transformed tissue was selected for resistance to kanamycin. Transformed progeny of the primary transformed regenerants were identified by germinating seed on standard Murashige-Skoog medium (GIBCO) with 100 $\mu \mathrm{g} / \mathrm{ml}$ kanamycin.

\section{Enzymatic assay of iaal}

Plant extracts were prepared by grinding tissue in liquid nitrogen, resuspending in $50 \mathrm{~mm}$ Tris (pH 8.0), $10 \mathrm{~mm} \beta$-mercaptoethanol, and $0.8 \mathrm{mM}$ phenazine methosulfate (PMSF), and then centrifuged at $12,000 \mathrm{~g}$ for $10 \mathrm{~min}$. The supernatant was then concentrated by two- to threefold by passage over a Centricon 30 filter prior to the assay. All steps were done at $4^{\circ} \mathrm{C}$. Assays of iaaL activity were done as described by Roberto et al. (1990) except that the reactions were run for $3.5 \mathrm{hr}$ at $28^{\circ} \mathrm{C}$ in the presence of $0.8 \mathrm{mM}$ PMSF. Reactions catalyzed by either bacterial or plant extracts containing iaaL activity were linear for at least $4 \mathrm{hr}$ (data not shown). Preparation of bacterial iaaL extracts derived from pMON686 and thin-layer chromatographs (TLCs) were also done as described except that the TLCs were not reacted with ninhydrin prior to fluorography (Roberto et al. 1990).

\section{Quantitation of IAA levels}

IAA was quantitated as described previously (Klee et al. 1987), but with the following modifications. Extracts were made from leaves, stems, and petioles of whole juvenile plants at about the six-node stage for the data in Table 1. Data in Table 3 were from leaves located 10 nodes up from the base of early flowering stage plants. Preparative separation of IAA and IAA-lysine from the crude plant extracts was achieved by reversed-phase HPLC on Zorbax (Dupont) C18. Quantitative analysis of IAA was done by sequential detection by absorbance $(260 \mathrm{~nm})$ and fluorescence (254 $\mathrm{nm}$ excitation, $340 \mathrm{~nm}$ emission) after isocratic separation on Vydac C8 in $12 \%$ methanol, $1.5 \%$ acetonitrile, and $0.05 \mathrm{M}$ acetate $(\mathrm{pH} 5.8)$. The ratio of the detectors' response was compared with that of an authentic standard of IAA (Sigma), and samples with detectors' ratios different from the standard were discarded from the analysis. An internal standard of $2-{ }^{14} \mathrm{C}$-IAA was added to each sample after extraction to measure recovery (Hein et al. 1984). Recovery of IAA from samples averaged $42 \%$ for the data in Table 1 and $20 \%$ for the data in Table 3. Enzymatically synthesized ${ }^{3} \mathrm{H}$-labeled IAA-lysine was used as an external standard to determine the chromatographic retention time of the conjugates.

\section{Plant growth conditions}

All phenotypic observations were made on plants grown in a greenhouse. Plants were grown in MetroMix 350 soil /Grace Chemical Company/ with daily watering and weekly fertilization (Peters Peat-Lite Special 151617 at 500 ppm nitrogen).

\section{Growth measurements}

Measurements were made on a minimum of six and a maximum of eight plants of a given genotype to generate the mean values and standard deviations of Table 1 . Flowering time is the number of days from germination to anthesis of the first, most apically derived floral bud. Height is the length from the base to 
the apical node of the mature plant. Internode lengths were obtained by dividing the height of the plant by the total number of nodes present. The midrib length was measured from the base to the tip of the leaf.

\section{Stem sections}

Stem cross sections of $100-\mathrm{mm}$ thickness were prepared with a Vibratome series 1000 sectioning device /Technical Products International, St. Louis, MO.|. Sections were stained with toluidine blue or phloroglucinol and photographed through a Zeiss Photomicroscope III.

\section{Acknowledgments}

We thank Nancy Mathis for production of transgenic tobacco and Bill Schuler for plant maintenance. We also thank Carl Braun, J. Scott Elmer, Maud Hinchee, Rob Horsch, and Robb Fraley for critical analysis and suggestions. Charles Romano gratefully acknowledges support as a postdoctoral associate by Monsanto.

The publication costs of this article were defrayed in part by payment of page charges. This article must therefore be hereby marked "advertisement" in accordance with 18 USC section 1734 solely to indicate this fact.

\section{References}

Aloni, R. 1988. Vascular differentiation within the plant. In Vascular differentiation and plant growth regulators /ed. T.E. Timell), pp. 39-62. Springer-Verlag, Berlin anbd Heidelberg.

Aloni, R. and M.H. Zimmermann. 1983. The control of vessel size and density along the plant axis-A new hypothesis. Differentiation 24: 203-208.

Bandurski, R.S. 1984. Metabolism of indole-3-acetic acid. In The biosynthesis and metabolism of plant hormones (ed. A. Crozier and J.R. Hillman), pp. 183-200. Cambridge University Press, Cambridge.

Benfey, P.N. and N.-H. Chua. 1989. Regulated genes in transgenic plants. Science 244: 174-181.

Bialek, K. and J.D. Cohen. 1989. Free and conjugated indole3-acetic acid in developing bean seeds. Plant Physiol. 91: 775-779.

Bialek, K., W.J. Meudt, and J.D. Cohen. 1983. Indole-3-acetic acid (IAA) and IAA conjugates applied to bean stem sections: IAA content and the growth response. Plant Physiol. 73: $130-134$.

Cleland, R.E. 1987. Auxin and cell elongation. In Plant hor mones and their role in plant growth and development (ed. P.J. Davies|, pp. 132-148. Martinus Nijhoff, Dordrecht, Boston.

Cohen, J.D. and R.S. Bandurski. 1982. Chemistry and physiology of the bound auxins. Annu. Rev. Plant Physiol. 33: 403430.

Cohen, J.D. and K. Bialek. 1984. The biosynthesis of indole3 -acetic acid in higher plants. In The biosynthesis and metabolism of plant hormones (ed. A. Crozier and J.R. Hillman), pp. 165-181. Cambridge University Press, Cambridge.

Ditta, G., S. Stanfield, D. Corbin, and D. Helinski. 1980. Broad host range DNA cloning system for gram-negative bacteria: Construction of a gene bank of Rhizobium meliloti. Proc. Natl. Acad. Sci. 77: 7347-7351.

Estelle, M.A. and C. Somerville. 1987. Auxin-resistant mutants of Arabidopsis thaliana with an altered morphology. Mol. Gen. Genet. 206: 200-206.
Fraley, R., S. Rogers, and R. Horsch. 1986. Genetic transformation in higher plants. CRC Crit. Rev. Plant Sci. 4: 1-45.

Glass, N.L. and T. Kosuge. 1986. Cloning of the gene for indoleacetic acid-lysine synthetase from Pseudomonas syringae subsp. savastanoi. I. Bacteriol. 166: 598-602.

Hagen, G. 1987. The control of gene expression by auxin. In plant hormones and their role in plant growth and develop. ment (ed. P.J. Davies), pp. 149-163. Martinus Nijhoff, Boston.

Hangartner, R.P. and N.E. Good. 1981. Evidence that IAA conjugates are slow release sources of free IAA in plant tissues. Plant Physiol. 68: 1424-1427.

Hein, M., M. Brenner, and W. Brun. 1984. Concentrations of abscisic-acid and indole-3-acetic acid in soybean seeds during development. Plant Physiol. 76: 951-954.

Horsch, R.B., J.E. Fry, N.L. Hoffmann, M. Wallroth, D. Eichholtz, S.G. Rogers, and R.T. Fraley. 1985. A simple and general method for transferring genes into plants. Science 227: 1229-1231.

Hutzinger, O. and T. Kosuge. 1968a. 3-indole-acetyl-e-L-lysine, a new conjugate of 3 -indoleacetic acid produced by Pseudomonas savastanoi. In Biochemistry and physiology of plant growth substances (ed. F. Wightman and G. Setterfield), pp. 183-194. Runge, Ottawa.

. 1968b. Microbial synthesis and degradation of indole3-acetic acid. 11l. The isolation and characterization of indole-3-acetyl- $\epsilon$-L-lysine. Biochemistry 7: 601-605.

Jefferson, R.A., T.A. Kavanagh, and M.W. Bevan. 1987. GUS fusions: $\beta$-Glucuronidase as a sensitive and versatile gene fusion marker in higher plants. EMBO J. 6: 3901-3907.

Kaufman, P.B. and I. Song. 1987. Hormones and the orientation of growth. In Plant hormones and their role in plant growth and development (ed. P.J. Davies), pp. 375-392. Martinus Nijhoff, Boston.

Kelly, M. and K. Bradford. 1986. Insensitivity of the diageotro pica tomato mutant to auxin. Plant Physiol. 82: 713-717.

Kunkel, T.A. 1985. Rapid and efficient site-specific mutagenesis without phenotypic selection. Proc. Natl. Acad. Sci. 82: 488-492.

Klee, H.J., R.B. Horsch, M.A. Hinchee, M.B. Hein, and N.L. Hoffmann. 1987. The effects of overproduction of two Agrobacterium tumefaciens T-DNA auxin biosynthetic gene products in transgenic petunia plants. Genes \& Dev. 1: 8696.

Lincoln, C., J.H. Britton, and M.A. Estelle. 1990. Growth and development of the axr1 mutants of Arabidopsis. Plant Cell 2: 1071-1080.

Medford, J.I., R. Horgan, Z. El-Sawi, and H.J. Klee. 1989. Alterations of endogenous cytokinins in transgenic plants using a chimeric isopentenyl transferase gene. Plant Cell 4: 403413.

Montague, M.J., R.K. Enns, N.R. Siegel, and E.J. Jaworski. 1981. Inhibition of 2,4-dichlorophenoxyacetic acid conjugation to amino acids by treatment of cultured soybean cells with cytokinins. Plant Physiol. 67: 701-704.

Napoli, C., C. Lemieux, and R. Jorgensen. 1990. Introduction of a chimeric chalcone synthase gene into petunia results in reversible co-suppression of the homologous genes in trans. Plant Cell 2: 279-289.

Roberto, F.F., H.J. Klee, F. White, R. Nordeen, and T. Kosuge. 1990. Expression and fine structure of the gene encoding N-G-indole-3-acetyl-L-lysine synthetase from Pseudomonas savastanoi. Proc. Natl. Acad. Sci. 87: 5797-5801.

Rogers, S.G., H.J. Klee, R.B. Horsch, and R.T. Fraley. 1987. Improved vectors for plant transformation: Expression cassette vectors and new selectable markers. Methods Enzymol. 
153: 253-277.

Schmülling, T., J. Schell, and A. Spena. 1988. Single genes from Agrobacterium rhizogenes influence plant development. EMBO I. 7: 2621-2629.

Schneider, E.A. and F. Wightman. 1978. Auxins. In Phytohormones and related compounds-A comprehensive treatise, (ed. D.D. Letham, P.B. Goodwin, and T.J. Higgins), vol. 1, pp. 29-105. Elsevier/North Holland Biomedical Press, New York.

Sinkar, V.P., F. Pythoud, F.F. White, E.W. Nester, and M.P. Gordon. 1988. rolA locus of the Ri plasmid directs developmental abnormalities in transgenic tobacco plants. Genes of Dev. 2: 688-697.

Skoog, F. and C.O. Miller. 1957. Chemical regulation of growth and organ formation in plant tissues cultured $\mathrm{n}$ vitro. Symp. Soc. Exp. Biol. 11: 188-231.

Tamas, I.A. 1987. Hormonal regulation of apical dominance. In Plant hormones and their roles in plant growth and development (ed. P.J. Davies), pp. 349-410. Martinus Nijhoff, Boston.

Thimann, K.V. 1963. Plant growth substances; past, present, and future. Annu. Rev. Plant Physiol. 14: 1-18.

Thimann, K.V. and F. Skoog. 1934. On the inhibition of bud development and other functions of growth substance in $\mathrm{Vi}$ cia faba. Proc. R. Soc. B114: 317-339.

Trewavas, A.J. 1982. Growth substance sensitivity: The limiting factor in plant development. Physiol. Plant. 55: 60-72.

van der Krol, A.R., L.A. Mur, M. Beld, J.N.M. Mol, and A.R. Stuitje. 1990. Flavanoid genes in petunia; addition of a limited number of gene copies may lead to a supression of gene expression. Plant Cell 2: 291-299.

Venis, M.A. 1972. Auxin-induced conjugation systems in peas. Plant Physiol. 49: 24-27. 


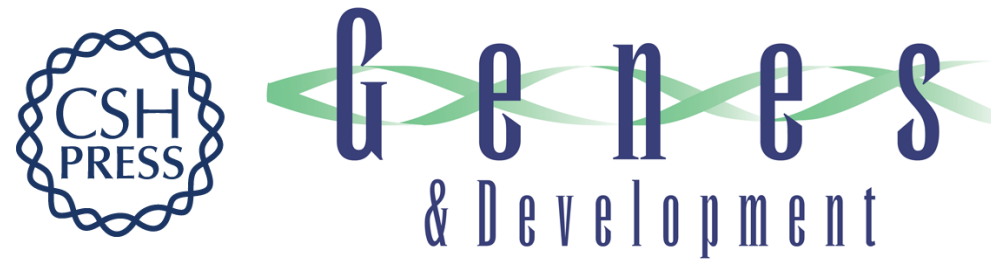

\section{Inactivation of auxin in tobacco transformed with the indoleacetic acid-lysine synthetase gene of Pseudomonas savastanoi.}

C P Romano, M B Hein and $\mathrm{H} J$ Klee

Genes Dev. 1991, 5:

Access the most recent version at doi:10.1101/gad.5.3.438

References This article cites 31 articles, 18 of which can be accessed free at:

http://genesdev.cshlp.org/content/5/3/438.full.html\#ref-list-1

License

Email Alerting

Service

Receive free email alerts when new articles cite this article - sign up in the box at the top right corner of the article or click here.

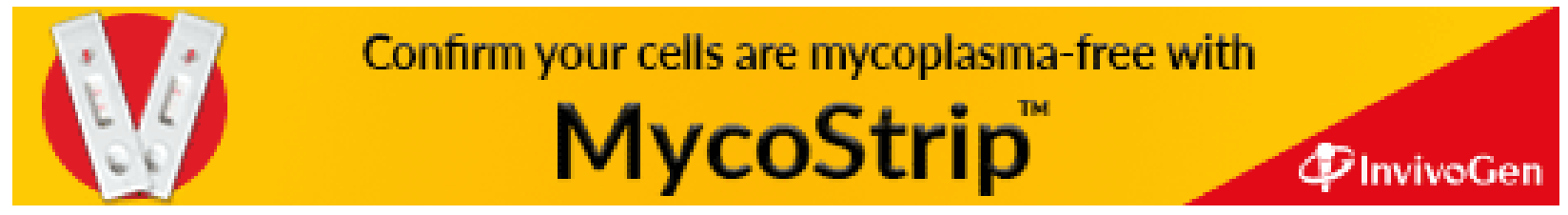

